\title{
Optimising long-term graft survival: establishing the benefit of targeting B lymphocytes
}

\author{
Authors: Kin Yee Shiu ${ }^{A}$ and Anthony Dorling ${ }^{\mathrm{B}}$
}

\begin{abstract}
Kidney transplants do not last for the natural lifespan of most recipients. Of the reasons why transplants fail, damage by the immune system is the commonest cause. Understanding how the immune system recognises transplanted organs has increased significantly in recent years, but there is little insight into how organs are damaged, and no still no way of suppressing immunemediated damage without exposing patients to the detrimental effects of long-term immunosuppression. In this article, we review the role of antibodies and $B$ cells in immune-mediated damage of kidney transplants, and discuss the potential for manipulation of B cells to improve clinical outcomes.
\end{abstract}

KEYWORDS: Transplantation, kidney, chronic rejection, B lymphocytes

\section{Introduction}

Kidney transplantation is the gold standard of treatment for patients with kidney failure, with better patient survival and quality of life compared with being on dialysis. However, many patients lose graft function during their lifetime and eventually return to dialysis. Up to $5 \%$ of the transplant population lose their graft in this manner each year, or approximately 700 patients in the UK. The most common cause for this premature graft failure is damage by the immune system. In order to address this problem, we need a better understanding of the immunological mechanisms that cause chronic damage.

\section{Alloantibodies and graft loss}

Although the pathogenesis of chronic graft dysfunction is multifactorial, ${ }^{1}$ immunological mechanisms are the major underlying problem in most kidney transplant recipients. There is now widespread recognition of the characteristic

Authors: ${ }^{\text {A }}$ consultant in nephrology and acute medicine, Royal Free London NHS Foundation Trust, London, UK; B professor of transplant inflammation and repair, MRC Centre for Transplantation, King's College London, and honorary consultant nephrologist, Guy's and St Thomas' NHS Foundation Trust, London, UK features of immune-mediated pathology seen on the transplant biopsy in most of these cases (Table 1). These features are strongly associated with the development of antibodies (Ab) directed against donor antigens (donor-specific Ab (DSA)), most commonly directed against human leukocyte antigens (HLA). These Ab, present in $20 \%$ of transplant patients, indicate that the recipient's immune system has been sensitised to HLA.

Both DSA and HLA-specific Ab (HLA Ab) are useful predictive biomarkers for an increased risk of graft failure. Graft failure rates 5 years after testing are under $20 \%$ in patients without HLA Ab, compared to approximately $50 \%$ in those with DSA and 30-40\% in patients with HLA Ab without DSA. ${ }^{2}$ The DSA or HLA Ab can appear many years before graft failure, during which time most patients develop graft dysfunction, manifesting most commonly as a slowly rising creatinine level and the development of proteinuria. A vast amount of experimental evidence supports the concept that these $\mathrm{Ab}$ are directly injurious to the graft, once bound to donor endothelium, via both complement-dependent and complement-independent mechanisms. Not surprisingly, complement-fixing $\mathrm{Ab}$ are associated with higher rates of acute and chronic rejection ${ }^{3}$ and are associated with positive $\mathrm{C} 4 \mathrm{~d}$ staining (a by-product of the activation of complement) on biopsies. ${ }^{4}$ These combinations of Ab, C4d and histopathological and/or molecular evidence of endothelial injury are now used together to diagnose 'antibody-mediated rejection' (AMR), ${ }^{5}$ a terminology reflecting the causative role that $\mathrm{Ab}$ are thought to play. However, while Abs certainly cause acute AMR, the evidence that they are the sole cause of 'chronic AMR' is substantially weaker.

\section{AlloAb and graft survival}

Approximately half of cases of chronic AMR diagnosed on transplant biopsy are negative for C4d and HLA $\mathrm{Ab}^{6}$ and acute rejection frequently precedes the development of DSA, ${ }^{7}$ both of which are consistent with the concept that other mechanisms contribute to graft dysfunction. Furthermore, many grafts continue to have stable function despite the presence of DSA/HLA Ab, consistent with the concept that $\mathrm{Ab}$ alone is insufficient in many cases to cause transplant dysfunction.

Another way of thinking of this is that some transplanted kidneys become resistant to AMR. This is called 'transplant 
1. Histological features of chronic, active AMR:

> transplant glomerulopathy - duplication of glomerular basement membrane which causes a 'double contour' appearance on light microscopy

$>$ transplant arteriopathy - fibrotic arterial intimal thickening

$>$ peritubular basement membrane multilayering

$>$ IFTA.

2. Evidence of current/recent $A b$ interaction with vascular endothelium:

$>$ linear C4d in peritubular capillaries

> moderate or severe microvascular inflammation (a feature of acute AMR) with increased inflammatory cells in glomeruli (transplant glomerulitis) or peritubular capillaries (peritubular capillaritis)

> endothelial injury detected through expression of gene transcripts.

3. Detectable donor-specific Ab (HLA or non-HLA):

$>$ may be historical (any time post-transplant) and not concurrent.

In addition, other features of acute AMR may be present: arteritis; acute thrombotic microangiopathy; or acute tubular injury. Lack of acute, active features and/or Ab at the time of the biopsy is termed chronic AMR, without the 'active' designation.

$\mathrm{Ab}=$ antibody; $\mathrm{AMR}=$ antibody-mediated rejection; HLA = human leukocyte antigens; IFTA = interstitial fibrosis and tubular atrophy.

accommodation' and was originally described in $\mathrm{ABO}-$ incompatible (ABOi) transplant recipients, ${ }^{8}$ but has also been seen in response to HLA-Ab. ${ }^{9}$ It is rarely described in non-ABOi recipients, but as a concept it may be relevant when trying to understand how patients with DSA or HLA Ab, particularly those with low-titre $\mathrm{Ab}$, maintain good transplant function; some investigators have proposed that it may be more widespread than acknowledged. ${ }^{10}$

\section{In chronic $\mathrm{AMR}, \mathrm{Ab}$ alone are insufficient and cellular mechanisms may play an important role}

In animal models of transplantation, alloAb alone are frequently insufficient to induce AMR, particularly in the absence of $\mathrm{T}$ and $\mathrm{B}$ cells, and in some rodent models, transfer of hyperimmune serum can even enhance graft survival. In rodent models of chronic AMR, transplant arteriosclerosis, the classical lesion of chronic rejection, can be mediated independently by any one of three mechanisms, including DSA, T cells and natural killer cells. ${ }^{11,12}$

T cells recognise graft major histocompatibility complex (MHC) antigens either via the 'direct' pathway (where intact MHC is recognised on donor cells) or the 'indirect' pathway (where CD4+ T cells recognise processed alloantigen presented as a peptide within self-MHC molecules on recipient antigen-presenting cells). T cells capable of indirect allorecognition persist for many years post-transplantation and are able to orchestrate rejection via several mechanisms including production of cytokines such as interferon (IFN) $\gamma$ by monocytes/macrophages in delayed-type hypersensitivity responses; promotion of cytotoxic CD4+ and CD8+ T cells; and through production of $\mathrm{Ab}$ via interactions with $\mathrm{B}$ cells. ${ }^{13}$ Detectable 'indirect' CD4+ T cell anti-donor reactivity has been associated with chronic graft dysfunction in human cardiac $^{14}$ and renal transplant patients, ${ }^{15}$ whereas quiescence has been associated with graft stability. ${ }^{16}$

\section{The role of B lymphocytes in transplantation - lessons from autoimmune disease}

The production of $\mathrm{Ab}$ requires a reciprocal interaction between antigen-specific $\mathrm{CD} 4+\mathrm{T}$ cells recognising processed antigen and HLA on antigen-specific B cells. Thus, in transplant patients with HLA-specific DSA, the presence of DSA is proof of previous interactions between $\mathrm{CD} 4+\mathrm{T}$ cells recognising donor HLA via the indirect pathway on donor-HLA-specific recipient $\mathrm{B}$ cells. As mentioned above, these 'indirect' T cells can mediate rejection via several effector pathways, but an important question is whether continuous interaction with antigenspecific memory B cells is involved in the maintenance of these effector responses.

Memory B cells play a key role in the development of $\mathrm{T}$ cell memory, as they are highly efficient at stimulating T-cell clonal expansion and proliferation, effective at up to $10^{4}$ times lower concentrations of antigen than either macrophages or nonspecific B cells; see Lanzavecchia et al. ${ }^{17}$ In contrast to naïve and activated B cells, their survival is not dependent on the continued presence of antigen, and they have a lower threshold for activation. On re-exposure to antigen they rapidly expand and differentiate into short-lived antibody-producing plasma cells, leading to a burst of Ab production. Memory B cells survive long-term, and differ significantly from plasma cells in that they reside in lymphoid tissue and do not require B cell activating factor (BAFF) or a proliferation inducing ligand (APRIL) survival factors. B cell memory is maintained by the survival of these antigen-specific cells, even when Ab levels are undetectable or low.

B-cell depletion using the anti-CD20 monoclonal antibody, rituximab, has been remarkably effective in treating patients with autoimmune disease, improving clinical and radiological outcomes in rheumatoid arthritis (RA), systemic lupus erythematosus (SLE) and multiple sclerosis (MS). The mechanism by which this occurs is incompletely 
understood, but one model is that these diseases arise due to a loss of tolerance to self-antigens, leading to a 'vicious cycle' of presentation of autoantigen by B cells to CD4+ T cells and resulting in reciprocal expansion of $\mathrm{T}$ - and $\mathrm{B}$-cell effector and memory responses, which includes differentiation into plasma cells and autoantibody production. ${ }^{18}$ Rituximab, by depleting $\mathrm{B}$ cells, breaks this cycle, as evidenced by the accompanying reductions in $\mathrm{T}$ - and $\mathrm{B}$-cell numbers in joint (in RA) or cerebrospinal fluid (in MS).

The effectiveness of rituximab highlights the crucial role that memory B cells play in sustaining effector mechanisms in these diseases. This is supported by some animal models of autoimmunity, in which tolerance is broken by vaccination with autoantigen, following which B-cell depletion, performed after the onset of disease, significantly reduces disease severity. ${ }^{19}$ Paradoxically, if B-cell depletion is performed prior to the initiation of disease in these animal models, disease severity is increased, suggesting that B cells play a role in sustaining tolerance to self. ${ }^{20}$ This is consistent with studies in which adoptive transfer of interleukin (IL)-10-producing B cells reduces disease severity in mouse models of MS and RA, possibly through the promotion of regulatory CD4+ T cells, ${ }^{18,21}$ and with clinical observations after rituximab administration to patients with autoimmune disease. A feature of prolonged remission in these patients is a loss of memory B-cell subsets, and the return of immature, transitional $\mathrm{B}$ cells, which are

\section{Cellular and antibody-mediated rejection: vicious cycle of $\mathrm{T}$ - and B-cell activation}

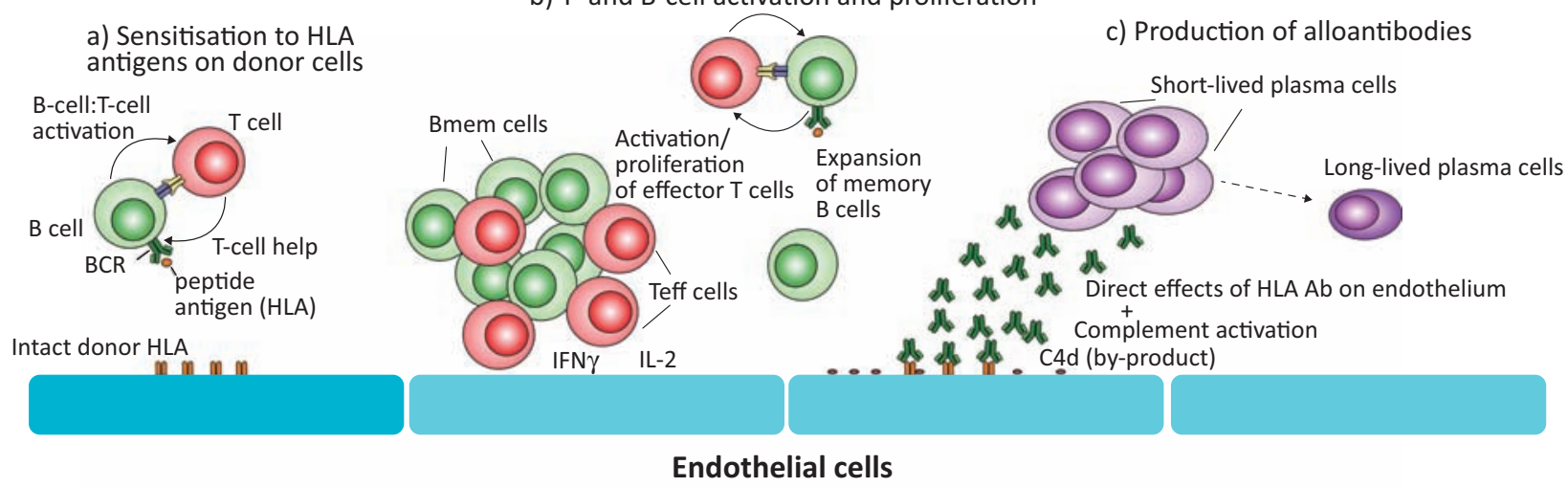

Tolerance and accommodation: B-cell depletion
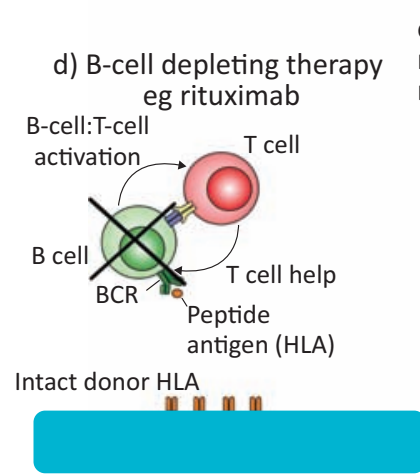

e) Effector T cells no longer activated nor expand
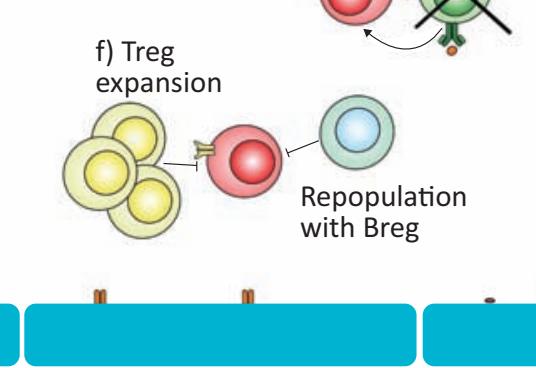

Endothelial cells g) Ongoing Ab production but without active inflammation

Fig 1. The role of $B$ cells in driving graft rejection in an $A b$-independent manner and the potential benefit of targeting $B$ cells. The pathogenesis of chronic antibody-mediated rejection is not fully understood, and the widely-held conception that donor-specific Ab, often specific for HLA, underlie the graft damage, is likely to be oversimplistic. Based on the available data, and experience from the effects of treating autoimmune disease with B-cell depletion, we suggest a model in which (a) sensitisation of T and B cells to graft antigens leads to a vicious cycle of (b) activation and expansion of both Teff and Bmem. (c) CD4+ effector T cells produce high levels of pro-inflammatory cytokines including IFN $\gamma$ and IL-2, in a delayed-type hypersensitivity response, which can induce direct damage. Simultaneously, there is a rapid expansion of short-lived plasma cells that may produce a burst of high levels of Ab directed at HLA, which can cause direct damage to endothelial cells as well as indirect effects through complement activation and antibody-dependent cell-mediated cytotoxicity. (d) When B-cell-depleting therapy is administered this vicious cycle is broken. (e) The activation of T cells is impaired, with reductions in the number and function of memory T cells. (f) This environment may promote the development of Tregs, and as transitional and naïve B cells repopulate from the bone marrow these are likely to further promote the development of tolerance to donor antigens, as well as cellular regulatory pathways. (g) Ab production may continue, as long-lived plasma cells can reside for many years, but in the absence of activated B cells contributing to a plasma cell pool, this is likely to be at low levels that can further promote the development of accommodation. $\mathrm{Ab}=$ antibody; $\mathrm{BCR}=\mathrm{B}$-cell receptor; $\mathrm{Bmem}=$ memory $\mathrm{B}$ cells; $\mathrm{HLA}=$ human leukocyte antigen; IFN = interferon; IL = interleukin; Teff = T effector cells; Treg = regulatory T cells. 
thought to re-establish tolerance by virtue of their predominant IL-10 secretion and regulatory properties. Indeed, there is some evidence that these types of $\mathrm{B}$ cells are dysfunctional in patients with SLE, suggesting they may be involved in the initial loss of self-tolerance. $^{22}$

In the transplantation arena, B cells have also been associated with operational tolerance and graft stability, ${ }^{23,24}$ suggesting that regulatory B cells may play a role in controlling alloimmunity. Recently, Nouel et al reported a reduction in the number and functional activity of transitional B cells in patients with chronic AMR, ${ }^{25}$ and Cherukuri et al reported associations between chronic rejection and a shift towards pro-inflammatory cytokine secretion by transitional B cells, compared with the predominant IL-10 secretion seen by transitional B cells in patients with stable graft function. ${ }^{26}$

\section{Optimising graft survival through B-cell depletion}

Highly sensitised transplant recipients, ie those with HLA $\mathrm{Ab}$, are at the greatest risk of acute and chronic rejection. All these patients have memory $\mathrm{B}$ and $\mathrm{T}$ cells specific for allogeneic HLA, akin to patients with autoimmune disease who have memory cells specific for autoantigens. B-cell depletion improves graft outcomes after transplantation into sensitised animals by lowering Ab levels and impairing T-cell activation and proliferation, reducing rates of acute cellular rejection and delaying or inhibiting development of chronic AMR. ${ }^{27}$ Rituximab has also been successfully used in induction protocols for highly sensitised transplant recipients (in sensitised recipients, this is not the same as deleting B cells prior to disease induction in immunologically-naïve animals) and is also frequently used in combination with intravenous immunoglobulins for the rescue treatment of acute AMR. ${ }^{28}$ Studies have been limited by small patient numbers and uncontrolled design, although a recent randomised controlled trial comparing IVIG + placebo and IVIG + rituximab for induction therapy of highly-sensitised patients reported a significant benefit from rituximab treatment, and was discontinued early due to a high rate of severe acute AMR in the placebo arm. ${ }^{29}$

Its role in the treatment of chronic AMR remains unclear, with small numbers of patients reported as benefiting from $B$ cell-depleting treatment in uncontrolled studies. ${ }^{30}$ The focus of our work has been trying to understand how B cells interact with $\mathrm{T}$ cells in patients with chronic AMR, diagnosed on the basis of either protocol or 'for cause' transplant biopsies. In approximately $20 \%$ of patients, we have demonstrated detectable IFN $\gamma$ production (using Enzyme Linked ImmunoSPOT assay) by CD4+ T cells activated via indirect allorecognition, indicating that $\mathrm{B}$ cells are important for processing and presenting donor antigens. In a further $20 \%$, this B-dependent reactivity is only revealed by depletion of CD25+ T lymphocytes and these cells have a putative regulatory phenotype by flow cytometry. Finally, in another $20 \%$, IFN $\gamma$ production is revealed by depletion of CD19+ cells, suggesting that B cells in these individuals are behaving as functional suppressor cells, a finding supported by preliminary analysis of how B cells from these individuals behave after polyclonal stimulation (they tend to make IL-10). Interestingly, we have not been able to find any correlations between this ELISPOT pattern and either the number or phenotype of transitional B cells in these patients. Our provisional conclusions from all this work are that B cells play a significant role in indirect alloantigen-specific donor T-cell reactivity in some patients with chronic AMR, but there is active suppression of T-cell immunity by distinct phenotypes of $\mathrm{T}$ and B cells in approximately $40 \%$ of patients, suggesting a complex heterogeneity of immune-mediated graft dysfunction in these patients (Fig 1).

These data suggest that rituximab might be effective at disrupting on-going cellular immune responses in some individuals with chronic AMR, but might have adverse effects in others (ie those with putative regulatory B cells suppressing alloreactivity). Therefore, to address the role of rituximab in chronic AMR, we are conducting a randomised, controlled trial (RituxiCAN-C4 clinicaltrials.gov identifier NCT00476164). The objective is to test whether rituximab can stabilise kidney function (reduce $\triangle \mathrm{eGFR}$ ) in patients with biopsy-proven chronic AMR who fail to respond to optimised oral immunosuppressive therapy. After a run-in period on optimised oral therapy, 30 patients who fail to respond will be randomly allocated to rituximab or control (no rituximab) arms. Those responding to optimised oral immunosuppression (or not consenting to randomisation) enter a three-year observational phase to inform the natural history of chronic AMR. To date, 58 have been recruited and 20 randomised. More than 30 have completed three years of follow-up or suffered graft failure. The trial is based on an estimate that $11 / 15$ patients given rituximab and $4 / 15$ controls will stabilise within 5 months of randomisation. An interim analysis of outcomes will be performed in late 2014 .

\section{Conclusion}

The production of $\mathrm{Ab}$ is a product of a reciprocal interaction between T and B cells that involves important 'cross-talk' through which the $\mathrm{T}$ cells provide crucial activation signals for the B cells, which may either become Ab-producing cells or antigen-specific memory B cells. Antigen-specific B cells provide potent activation and proliferation signals to $\mathrm{T}$ cells, thus leading to $\mathrm{T}$ cell effector mechanisms which can themselves cause injury, and potentially drive graft rejection. These immune effector processes underpin the damage that occurs in transplanted kidneys suffering from chronic AMR, which has emerged as the commonest cause of premature allograft failure. The critical questions we are asking relate to the importance of the cellular effector responses, compared with $\mathrm{Ab}$, and whether targeting memory B cells, using rituximab, can prevent the progressive functional decline often seen in these patients. Improved understanding of the precise mechanisms involved in chronic AMR, and the ability to distinguish which mechanism is active in individual transplant recipients, should lead to the development of rational, safe and effective treatments to prolong graft survival.

\section{Acknowledgements}

The authors acknowledge that this research was supported by the National Institute for Health Research (NIHR) Biomedical Research Centre based at Guy's and St Thomas' NHS Foundation Trust and King's College London. The views expressed are those of the author(s) 
and not necessarily those of the NHS, the NIHR or the Department of Health. The work referred to here was supported by MRC grants G84/6713 and G0801965, Roche Organ Transplant Research Foundation Award ROTRF 53331024, Novartis Pharmaceuticals nonpromotional grant TRA10-087 and KRUK grant RP3/2011.

\section{References}

1 Chapman JR, O'Connell PJ, Nankivell BJ. Chronic renal allograft dysfunction. J Am Soc Nephrol 2005;16:3015-26.

2 Lachmann N, Terasaki PI, Budde K et al. Anti-human leukocyte antigen and donor-specific antibodies detected by luminex posttransplant serve as biomarkers for chronic rejection of renal allografts. Transplantation 2009;87:1505-13.

3 Loupy A, Lefaucheur C, Vernerey D et al. Complement-binding anti-HLA antibodies and kidney-allograft survival. $N$ Engl J Med 2013;369:1215-26.

4 Feucht HE, Schneeberger H, Hillebrand G et al. Capillary deposition of $\mathrm{C} 4 \mathrm{~d}$ complement fragment and early renal graft loss. Kidney Int 1993;43:1333-8.

5 Haas M, Sis B, Racusen LC et al. Banff 2013 meeting report: inclusion of c4d-negative antibody-mediated rejection and antibodyassociated arterial lesions. Am J Transplant 2014;14:272-83.

6 Sis B, Campbell PM, Mueller T et al. Transplant glomerulopathy, late antibody-mediated rejection and the $\mathrm{ABCD}$ tetrad in kidney allograft biopsies for cause. Am J Transplant 2007;7:1743-52.

7 Wiebe C, Gibson IW, Blydt-Hansen TD et al. Evolution and clinical pathologic correlations of de novo donor-specific HLA antibody post kidney transplant. Am J Transplant 2012;12:1157-67.

8 Dorling A. Transplant accommodation - are the lessons learned from xenotransplantation pertinent for clinical allotransplantation? Am J Transplant 2012;12:545-53.

9 Salama A, Delikouras A, Pusey CD et al. Transplant accommodation in highly sensitised patients: A potential role for $\mathrm{Bcl}-\mathrm{xL}$ and alloantibody. Am J Transplant 2001;1:260-9.

10 Lynch RJ, Platt JL. Accommodation in renal transplantation: unanswered questions. Curr Opin Organ Transplant 2010;15:481-5.

11 Uehara S, Chase CM, Cornell LD et al. Chronic cardiac transplant arteriopathy in mice: relationship of alloantibody, C4d deposition and neointimal fibrosis. Am J Transplant 2007;7:57-65.

12 Hirohashi T, Chase CM, Della Pelle P et al. A novel pathway of chronic allograft rejection mediated by NK cells and alloantibody. Am J Transplant 2012;12:313-21.

13 Rocha PN, Plumb TJ, SD Crowley SD et al. Effector mechanisms in transplant rejection. Immunol Rev 2003;196:51-64.

14 Hornick PI, Mason PD, Baker RJ et al. Significant frequencies of T cells with indirect anti-donor specificity in heart graft recipients with chronic rejection. Circulation 2000;101:2405-10.

15 Baker RJ, Hernandez-Fuentes MP, Brookes PA et al. Loss of direct and maintenance of indirect alloresponses in renal allograft recipients: implications for the pathogenesis of chronic allograft nephropathy. J Immunol 2001;167:7199-206.
16 Salama AD, Najafian N, Clarkson MR et al. Regulatory CD25+ T cells in human kidney transplant recipients. J Am Soc Nephrol 2003;14:1643-51.

17 Lanzavecchia A, Sallusto F. Human B cell memory. Curr Opin Immunol 2009;21:298-304.

18 Lund FE, Randall TD. Effector and regulatory B cells: modulators of CD4+ T cell immunity. Nat Rev Immunol 2010;10:236-47.

19 Bouaziz JD, Yanaba K, Venturi GM et al. Therapeutic B cell depletion impairs adaptive and autoreactive CD4+ T cell activation in mice. Proc Natl Acad Sci U S A 2007;104:20878-83.

20 Matsushita T, Yanaba K, Bouaziz JD et al. Regulatory B cells inhibit EAE initiation in mice while other $\mathrm{B}$ cells promote disease progression. J Clin Invest 2008;118:3420-30.

21 Carter NA, Vasconcellos R, Rosser EC et al. Mice lacking endogenous IL-10-producing regulatory $\mathrm{B}$ cells develop exacerbated disease and present with an increased frequency of Th1/Th17 but a decrease in regulatory T cells. J Immunol 2011;186:5569-79.

22 Blair PA, Norena LY, Flores-Borja F et al. CD19(+)CD24(hi) CD38(hi) B cells exhibit regulatory capacity in healthy individuals but are functionally impaired in systemic Lupus Erythematosus patients. Immunity 2010;32:129-40.

23 Sagoo P, Perucha E, Sawitzki B et al. Development of a cross-platform biomarker signature to detect renal transplant tolerance in humans. J Clin Invest 2010;120:1848-61.

24 Newell KA, Asare A, Kirk AD et al. Identification of a B cell signature associated with renal transplant tolerance in humans. J Clin Invest 2010;120:1836-47.

25 Nouel A, Segalen I, Jamin C et al. B cells display an abnormal distribution and an impaired suppressive function in patients with chronic antibody-mediated rejection. Kidney Int 2014;85:590-9.

26 Cherukuri A, Rothstein DM, Clark B et al. Immunologic human renal allograft injury associates with an altered IL-10/TNF-alpha expression ratio in regulatory B cells. J Am Soc Nephrol 2014;25:1575-85.

27 Levesque V, Bardwell PD, Shimizu I et al. B-cell-dependent memory T cells impede nonmyeloablative mixed chimerism induction in presensitized mice. Am J Transplant 2011;11:2322-31.

28 Barnett AN, Hadjianastassiou VG, and Mamode N. Rituximab in renal transplantation. Transpl Int 2013;26:563-75.

29 Vo AA, Choi J, Cisneros K et al. Benefits of rituximab combined with intravenous immunoglobulin for desensitisation in kidney transplant recipients. Transplantation 2014;98:312-9.

30 Kahwaji J, Najjar R, Kancherla D et al. Histopathologic features of transplant glomerulopathy associated with response to therapy with intravenous immune globulin and rituximab. Clin Transplant 2014;28:546-53.

Address for correspondence: Prof A Dorling, MRC Centre for Transplantation, King's College London, Guy's Hospital, London SE1 9RT, UK.

Email: anthony.dorling@kcl.ac.uk 